\title{
Evaluación Abreviada del Conocimiento Fonológico mediante la Prueba P.E.C.O.
}

\section{Short Assessment Test by Phonological Awareness P.E.C.O.}

\author{
José Luís Ramos Sánchez * \\ Ana Isabel González Contreras \\ Universidad de Extremadura - España
}

\begin{abstract}
El objetivo de este este trabajo es obtener una versión abreviada del conocimiento fonológico a partir de la prueba P.E.C.O. manteniendo una validez adecuada. Entendemos por conocimiento fonológico la habilidad para tomar conciencia y manipular los elementos más simples del lenguaje oral como son las sílabas (conocimiento silábico) y los fonemas (conocimiento fonémico). Un adecuado desarrollo del conocimiento fonológico junto con otras habilidades como la velocidad de denominación son fundamentales para la adquisición del principio alfabético, y por tanto para aprender a leer y a escribir en un idioma transparente como el español. Para llevar a cabo el estudio, se toma la muestra original de tipificación $(\mathrm{N}=240)$ formada por alumnos y alumnas de habla española de educación infantil de 5 años (mediana de edad de 5 años y 9 meses) y se utiliza el análisis de regresión múltiple (método de pasos sucesivos) para obtener un modelo de regresión que maximiza una predicción ajustada con nueve ítems. A partir de los resultados se obtuvo una versión abreviada y representativa de la prueba original, que contiene ítems variados en cuanto al tipo de tareas (identificación, adición y omisión) con garantías de predecir el rendimiento en conocimiento fonológico de los alumnos en tres niveles: alto, medio y bajo.
\end{abstract}

Palabras clave: Aprendizaje inicial de la lectoescritura; Análisis de regresión múltiple; Evaluación del conocimiento fonológico; Principio alfabético.

The objective of this work is to obtain an abridged version of phonological awareness from the test P.E.C.O. maintaining adequate validity. We understand phonemic awareness skills to become aware and manipulate the simplest elements of spoken language such as syllables (syllabic knowledge) and phonemes (phonemic awareness). Proper development of phonological awareness along with other skills such as naming speed are essential for the acquisition of the alphabetic principle, and thus to learn to read and write in a transparent language such as Spanish. To conduct the study, the original sample typing $(\mathrm{N}=240)$ formed by students from Spanishspeaking child education of 5 years (median age of 5 years and 9 months) is taken and used analysis multiple regression (stepwise method) to obtain a regression model that maximizes an adjusted prediction nine items. From the results an abbreviated and representative version of the original test, containing various items in the type of tasks (identification, addition and omission) guarantees to predict performance in phonological awareness of students in three levels was obtained: high, medium and low.

Keywords: Initial literacy learning; Multiple regression analysis; Evaluation of phonological awareness; Alphabetic principle.

\footnotetext{
*Contacto: jlramos@unex.es

Recibido: $\quad 18$ de abril de 2016

$1^{\text {a }}$ Evaluación: 25 de julio de 2016

Aceptado: $\quad 25$ de septiembre de 2016 


\section{Introducción}

El conocimiento fonológico o conciencia fonológica es la habilidad para tomar conciencia y manipular los elementos más simples del lenguaje oral como son las sílabas (conocimiento silábico) y los fonemas (conocimiento fonémico), y son numerosos los trabajos que demuestran la importancia de esta habilidad metalingüística, tanto para la adquisición de la lectoescritura en educación infantil como variable deficitaria y susceptible de refuerzo en el alumnado disléxico y con dificultades en el aprendizaje lectoescritor. Un buen ejemplo de ello son los trabajos ya clásicos de Sebastián y Maldonado (1987), Sánchez, Rueda y Orrantía (1989), Carrillo y Sánchez (1991, 1996), Domínguez (1992, 1996), Ortíz (1994), Jiménez y Ortiz (1995), Wagner, Torgesen y Rashotte (1994), Ramos (2000, 2004), Ramos y Cuadrado (2004), etc.

Se piensa que el conocimiento fonológico es crucial para llevar a cabo las Reglas de Conversión Grafema-Fonema (RCGF) que son necesarias durante la decodificación fonológica (Manis et al., 1999; Vellutino et al., 2004). Y se ha hecho mucho hincapié en demostrar cómo el trabajar con fonemas es una herramienta específica para la adquisición del principio alfabético (Byrne y Fielding-Barnsley, 1989; De Jong y Olson, 2004). Aunque también sabemos, que una vez que el alumno se ha iniciado en los procesos de decodificación, la influencia del conocimiento fonológico es menor, y la prueba de ello es que los coeficientes de correlación entre el conocimiento fonológico y el rendimiento lectoescritor disminuyen al año siguiente de iniciarse al alumnado en la lectoescritura (ver cuadros 8 y 9 de este trabajo).

Por estas evidencias, el conocimiento fonológico forma parte tanto del conjunto de variables en el proceso de evaluación como de la intervención en gran parte del alumnado con dificultades en los procesos fonológicos de lectura y escritura. La Prueba de Evaluación del Conocimiento Fonológica (PECO) constituye un ejemplo contrastado de evaluación del conocimiento fonológico y ha sido utilizada para la evaluación del alumnado y en investigaciones sobre el tema. Pero a veces las características de los sujetos (edad, nivel de atención, etc.) aconsejan, por un motivo u otro, brevedad en la aplicación.

PECO original es una prueba de aplicación individual compuesta por 30 ítems, y por la experiencia en la aplicación de la misma, el tiempo empleado es de 30 a 40 minutos aproximadamente. A veces un tiempo excesivo para algunos alumnos, especialmente para aquellos más predispuestos al cansancio y al abandono de la tarea: alumnado menor de seis años, alumnado con déficit de atención, etc. Y en otras ocasiones, la limitación de tiempo o de presupuesto dificulta la tarea de investigación.

Es cierto que, además del conocimiento fonológico, existen evidencias sobre otras variables que influyen en el aprendizaje inicial de lectura y escritura; por ejemplo, la velocidad de denominación o de nombrado, entendida como el tiempo empleado por el alumno en identificar un estímulo visual. En las tareas típicas de denominación rápida se pide nombrar en voz alta diversos estímulos (dibujos, letras, números o colores), tan rápido como sea posible y se toma como parámetro el tiempo total que se emplea para nombrarlos. Existe correlación entre la velocidad de denominación y el aprendizaje inicial de la lectoescritura en los niños con desarrollo normal (Cutting y Denckla, 2001; de Jong y Van der Leij, 1999; Kirby et al., 2003; Powell et al., 2007). 
El estudio realizado por Guzmán et al. (2004) demostró que el déficit de velocidad de denominación en niños con dificultades lectoras es causado por su déficit en el procesamiento fonológico, tomando la idea de que si las tareas de velocidad de denominación realizan un papel importante en la adquisición de representaciones ortográficas de las palabras, sus efectos se deben también considerar en medidas de exactitud y de velocidad. Los resultados demostraron que los alumnos con dificultades de adquisición en la lectura y déficit fonológico eran más lentos en las tareas de nombrar colores, dibujos, letras y números que los lectores normales de su misma edad.

Igualmente, López-Escribano (2007) confirma otros estudios en los que se afirma que la velocidad de denominación contribuye fuertemente a la velocidad de la lectura y al reconocimiento ortográfico. La velocidad de denominación afecta sobre todo a la realización de las tareas de la lectura que requieren una respuesta fluida. Su investigación confirma los resultados que asocian el reconocimiento ortográfico a las habilidades de la lectura.

Por su parte, Gómez et al. (2010) comprobaron que la denominación de letras predijo mejor la ejecución lectora e identificó correctamente a 63\% de los niños de 7 años en su estudio $(\mathrm{N}=121)$ que posteriormente presentaron dificultades en la velocidad para leer, la cual se ha considerado como el rasgo distintivo de la dislexia en español. Algunas tareas de conciencia fonológica contribuyeron a explicar la eficiencia y la comprensión lectora. Sin embargo, los niños con un doble déficit (menor velocidad de denominación y de conciencia fonológica) presentaron el peor rendimiento lector.

Esta conclusión tiene una gran importancia para la detección temprana de las dificultades de la lectoescritura, en el sentido de que sería necesario incluir la evaluación de la velocidad de denominación junto con el conocimiento fonológico al finalizar la educación infantil como predictores de las dificultades de lectura al inicio de la educación primaria o al finalizar la educación infantil.

Nuestro trabajo tiene como objetivo identificar los ítems más relevantes de PECO para predecir lo mejor posible el nivel de conocimiento fonológico del alumno en base a esta prueba. Por tanto, se pretende obtener una versión abreviada de PECO teniendo en cuenta la composición heterogénea del conocimiento fonológico, con objeto de disminuir el tiempo de evaluación manteniendo una adecuada calidad técnica.

\section{Fundamentación teórica}

El conocimiento fonológico es objeto de investigación desde hace más de tres décadas, y son numerosas las investigaciones que utilizan esta variable, tanto en el proceso de explicación causal y relacional de las dificultades lectoescritoras como en el proceso de intervención para mejorar su desarrollo o con la finalidad de prevenir dificultades. A modo de ejemplo, los trabajos de Trias, Cuadro y Costa (2009), Defior y Serrano (2011), Muñoz (2011), Bravo, Villalón y Orellana (2011), Leal y Suro (2012), Suárez, García y Cuetos (2013), Delgado y Sancho (2014), Feld (2014) y González, Cuetos, Vilar y Uceira (2015), son una pequeña muestra del interés actual sobre la importancia de disponer de instrumentos que evalúen el conocimiento fonológico.

Precisamente, dada la necesidad de que los profesionales de la educación y la psicología dispusieran de una prueba para evaluar el desarrollo del conocimiento fonológico, fue 
elaborada PECO de Ramos y Cuadrado (2006a), y consecuentemente también fueron elaborados materiales para la intervención (Ramos y Cuadrado, 2006b).

En la elaboración de PECO se tuvo en cuenta que el conocimiento fonológico no tiene una entidad homogénea, sino que depende de distintas variables relacionadas con la estructura de las palabras. Así, PECO fue elaborada ajustándose a los cuatro criterios que más se repiten en la evaluación del conocimiento fonológico. A saber, la unidad fonológica (sílabas y fonemas), el tipo de tarea (análisis y síntesis), la posición del segmento fonológico (inicial, medio y final) y la estructura silábica utilizada.

En cuanto a la unidad fonológica, identificamos tres niveles de conocimiento fonológico: palabras, sílabas y fonemas. Las unidades más importantes en la adquisición inicial de la lectoescritura son la fonémica y la silábica por este orden. Son más fáciles las tareas relacionadas con las palabras y las sílabas que con los fonemas.

Según el tipo de tarea, se identifican básicamente dos tipos: análisis y síntesis. Las tareas de análisis tienen como objetivo tomar conciencia y manipular unidades aisladas de las palabras tales como las de rima, identificación, aislamiento, recuento, omisión, sustitución e inversión. Las tareas de síntesis conllevan la habilidad para recomponer, recodificar o formar una palabra que se presenta dividida en partes. Este es el objetivo de las tareas de adición y unión de sílabas y/o fonemas. En cuanto a la dificultad, las tareas de rimas e identificación son más fáciles que las de omisión y adición; mientras que las tareas más complejas son las de inversión, que requieren un gran esfuerzo cognitivo no tan necesario para aprender a leer y escribir (Yopp, 1988).

La posición del segmento fonológico constituye otra fuente de variabilidad de las tareas, según sea al inicio, en el medio o al final de la palabra. Los resultados del trabajo de Domínguez y Clemente (1993) demuestran que la enseñanza de las habilidades de análisis de las palabras es igual de efectiva utilizando sonidos iniciales, medios o finales, puesto que el aprendizaje por parte de los alumnos en una de las posiciones se generaliza a otras posiciones de la palabra. Aunque admiten que es más fácil reconocer sílabas y fonemas en posición inicial que al final y ambas actividades son más fáciles que identificar sílabas o fonemas en el medio de las palabras. Mientras que, en las tareas de omisión y adición, es más sencillo operar con segmentos finales que iniciales, y ambas son más fáciles que las tareas con unidades mediales.

Finalmente, otra variable clasificatoria de las tareas de conocimiento fonológico es la referida al tipo de sílaba. No es lo mismo operar con grupos consonánticos complejos del tipo CCV (Consonante+Consonante+Vocal) o CCVC (ejemplo: brazo, tronco, ...) que utilizar sílabas simples, siendo estas últimas más fáciles de trabajar (CV - gato- o VC alto-) según demuestra la investigación de Treiman (1991).

\section{Método}

Como muestra utilizamos la original de la prueba compuesta por 240 alumnos $(n=120)$ y alumnas $(n=120)$ sin necesidades educativas especiales de educación infantil, de habla española, con un intervalo de edad entre 5 años y 4 meses y 6 años y 4 meses (mediana de 5 años y 9 meses), procedentes de centros educativos de zona urbana y rural, públicos y concertados. 
Para llevar a cabo el objetivo de investigación, se utiliza una metodología correlacional a través de la técnica del análisis de regresión múltiple del paquete estadístico SPSS (versión 18). Este tipo de análisis tiene dos finalidades en nuestro estudio. Por un lado, predecir la puntuación total en PECO; y por otro, identificar qué variables son las más relevantes en la predicción. Precisamente estas variables serán las que formen parte de la versión abreviada si se cumplen las condiciones de aplicación y la validación del modelo de regresión.

En cuanto a las variables, las predictoras (independientes) son los 30 ítems que conforman la prueba original y como variable criterio (dependiente) se utilizó la puntuación total en la prueba. Y en relación con el método para la introducción de las distintas variables en el análisis de regresión, se utiliza el de pasos sucesivos.

\section{Resultados}

Presentamos un resumen de la aplicación de la técnica del análisis de regresión lineal multivariada de SPSS (v.18), aunque por brevedad no se incluyen los resultados de las distintas etapas. En el cusdro 1 se muestran las nueve variables incluidas en el análisis, pero no se muestran las veintiuna variables excluidas. Estas variables o ítems se codifican utilizando los siguientes indicadores: las dos primeras letras hace referencia al tipo de tarea que el alumno debe realizar en el ítem (of: omisión de fonemas, af: adición de fonemas, if: identificación de fonemas, os: omisión de sílabas, as: adición de sílabas), el número que aparece a continuación se corresponde con el número del ítem en la prueba original y los ítems seleccionados son, por orden, los siguientes: 26, 17, 10, 13, 25, 6, 23, 20 y 14. La letra después del número es la posición del fonema o de la sílaba de la palabra-estímulo utilizada en la prueba ( $i=$ inicial, $f=$ final, $m=$ medial).

En el cuadro 1 comprobamos que los coeficientes de regresión van asociados a un valor de t significativo, y que los estadísticos de colinealidad, medido a través del coeficiente de tolerancia ( $\mathrm{T})$, se aproximan a 1. Este coeficiente nos permite considerar que existe una ausencia de multicolinealidad. Es decir, cada una de las variables predictoras es independiente del resto, con lo cual cada una de ellas aporta información relevante de cara a la validez del modelo predictivo.

Cuadro 1. Variables incluidas en el análisis

\begin{tabular}{lccc}
\hline \multicolumn{1}{c}{ Variables } & $\mathbf{B}$ & $\mathbf{t}$ & $\mathbf{T}$ \\
\hline Constante & 2,821 & $7,695^{*}$ & \\
of26i & 2,834 & $11,497^{*}$ & 0,804 \\
af17f & 2,261 & $8,390^{*}$ & 0,733 \\
if10m & 1,828 & $7,116^{*}$ & 0,738 \\
as13i & 1,759 & $6,415^{*}$ & 0,693 \\
os25m & 2,538 & $8,694^{*}$ & 0,844 \\
if6i & 2,325 & $6,409^{*}$ & 0,772 \\
os23i & 2,268 & $8,954^{*}$ & 0,775 \\
af20m & 1,624 & $6,112^{*}$ & 0,794 \\
as14i & 1,627 & $6,166^{*}$ & 0,692 \\
\hline
\end{tabular}

Notas: B: Coeficientes de regresión no estandardizados; t: Prueba t; T: Estadístico de colinealidad (tolerancia); (*): Significación de $\mathrm{t}<0,000$.

Fuente: Elaboración propia. 
Por otro lado, en el cuadro 2 observamos que con las nueve variables incluidas conseguimos un coeficiente de determinación corregido $\left(\mathrm{R}_{\mathrm{c}}{ }_{\mathrm{c}}\right)$ de 0,922 . Esto significa que el 92,2\% de la puntuación total de PECO es explicada por estas nueve variables que forman parte de la versión abreviada. Por su parte, y como es fácil de suponer, teniendo en cuenta el elevado valor del coeficiente de determinación corregido, el valor $\mathrm{F}$ de ANOVA $(216,843)$ es significativo, por tanto, los coeficientes de regresión obtenidos son significativos para la predicción.

Cuadro 2. Resumen del modelo

\begin{tabular}{cccccc}
\hline $\mathbf{R}$ & $\mathbf{R}^{2}$ & $\mathbf{R}^{2}$ CORREGIDA & ERROR TÍP. DE & DURBIN-WATSON & ANOVA F \\
\hline 0,962 & 0,926 & 0,922 & 1,700 & 1,883 & $216,843 \mathrm{p}<, 001$ \\
\hline
\end{tabular}

Fuente: Elaboración propia.

Además de la ausencia de colinealidad, otra de las condiciones de la aplicación del análisis de regresión es la comprobación de que la relación entre las variables sea lineal, para ello realizamos un estudio de los residuos tipificados en función de los valores pronosticados para la variable dependiente. Pues bien, podemos comprobar en el gráfico 1 cómo los residuos se distribuyen de manera aleatoria al ajustarse claramente a una nube de puntos sin apreciarse ninguna tendencia. Podemos demostrar que la relación entre las variables es lineal y con igualdad de varianzas (homoscedasticidad), y estas condiciones favorece la precisión de la predicción a partir del modelo de regresión. Otro dato a favor del modelo a partir del estudio de los residuos es la posible existencia de autocorrelación que se obtiene mediante el estadístico de Durbin-Watson $(1,883)$ (cuadro 2), y el hecho de que sea próximo a 2 certifica la inexistencia de autocorrelación de los residuos.

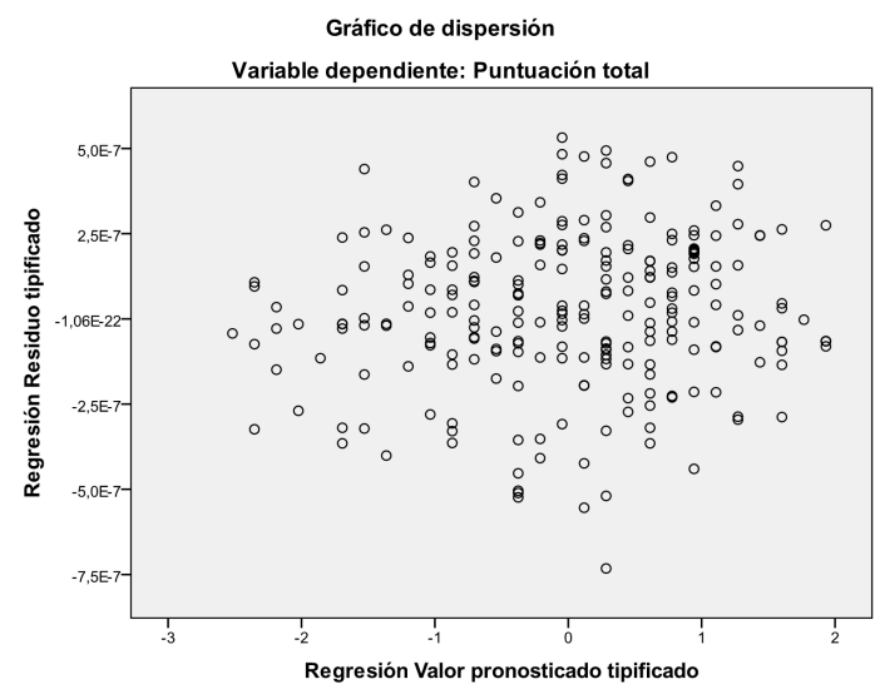

Gráfico 1. Análisis de residuos

Fuente: Elaboración propia.

Por tanto, teniendo en cuenta la evaluación del conocimiento fonológico a partir de los ítems seleccionados por el modelo de regresión, podríamos utilizar los siguientes datos descriptivos y de tipificación e interpretación del nivel de conocimiento fonológico (cuadros 3 y 4). Al ser 9 ítems, la puntuación máxima que se puede obtener es de 9 puntos. 
Cuadro 3. Datos descriptivos

\begin{tabular}{ccc}
\hline MEDIA & DESVIACIÓN TÍPICA & MEDIANA \\
\hline 4,78 & 2,188 & 5,00 \\
\hline
\end{tabular}

Fuente: Elaboración propia.

En la tabla de tipificación e interpretación de resultados se muestran las puntuaciones directas correspondientes a los valores cuartiles. Así, en el cuartil $1\left(Q_{1}\right)$ se sitúan los alumnos cuyo percentil es igual o inferior a 25; es decir, los que obtienen una puntuación directa de 3 puntos o menos, con lo cual consideramos que su nivel de conocimiento fonológico es BAJO, mientras que los que se sitúen en el cuartil 2 y $3\left(Q_{2}\right.$ y $\left.Q_{3}\right)$ serían respectivamente los niveles MEDIO y ALTO.

Cuadro 4. Tipificación e interpretación de los resultados en la aplicación abreviada

\begin{tabular}{lccc}
\hline & $\mathbf{Q}_{1}$ & $\mathbf{Q}_{2}$ & $\mathbf{Q}_{\mathbf{3}}$ \\
& CENTIL $\leq$ 25 & CENTIL ENTRE 26 Y 75 & CENTIL > 75 \\
\hline Nivel & Bajo & Medio & Alto \\
Punt. directa versión abreviada & 3 y menos & De 4 a 6 & 7 y más \\
\hline
\end{tabular}

Fuente: Elaboración propia.

Por otro lado, presentamos una forma sencilla de obtener los valores pronosticados en la prueba original a partir de la puntuación total en la prueba abreviada. La ecuación de regresión a partir de la variable predictora "Puntuación total Abreviada" (PtA) sería: $\Upsilon^{\prime}{ }_{P t}$ $=4,985+2,575^{*}$ PtA. Por ejemplo, a un sujeto que obtiene 5 puntos en la versión abreviada se pronostica una puntuación derivada de la fórmula $\mathrm{Y}_{\mathrm{Pt}}^{\prime}=4,985+2,575^{*}(5)=17,86$ (por redondeo 18).

En el cuadro 5 presentamos los principales estadísticos del análisis de regresión en la nueva versión abreviada. En este caso, y como es lógico, el valor de coeficiente de determinación ha disminuido en comparación con el del modelo de regresión múltiple, puesto que la predicción se realiza sobre un solo predictor, y por consiguiente el error típico de la estimación aumenta respecto al modelo lineal múltiple, que pasa de 1.700 a 2.264. En cuanto a las características del modelo de regresión simple, también observamos los óptimos valores obtenidos en el coeficiente de determinación, los valores de DurbinWatson (muy próximo a 2,00) y al elevado valor de F.

Cuadro 5. Resumen del modelo con el predictor PtA

\begin{tabular}{cccccc}
\hline $\mathbf{R}$ & $\mathbf{R}^{2}$ & $\mathbf{R}^{2}$ CORREGIDA & ERROR TÍP. & DURBIN-WATSON & ANOVA F \\
\hline 0,928 & 0,861 & 0,861 & 2,264 & 1,986 & $1479,73 \mathrm{p}<, 001$ \\
\hline
\end{tabular}

Fuente: Elaboración propia.

Las condiciones para la aplicación del modelo de regresión lineal simple se cumplen en su totalidad. Por un lado, no tiene sentido plantearse la existencia o no de multicolinealidad al existir una sola variable, y por otro lado, el análisis de los residuos (Gráfico 2) demuestran una distribución aleatoria, y por tanto, queda demostrada la linealidad y homoscedasticidad del modelo. 


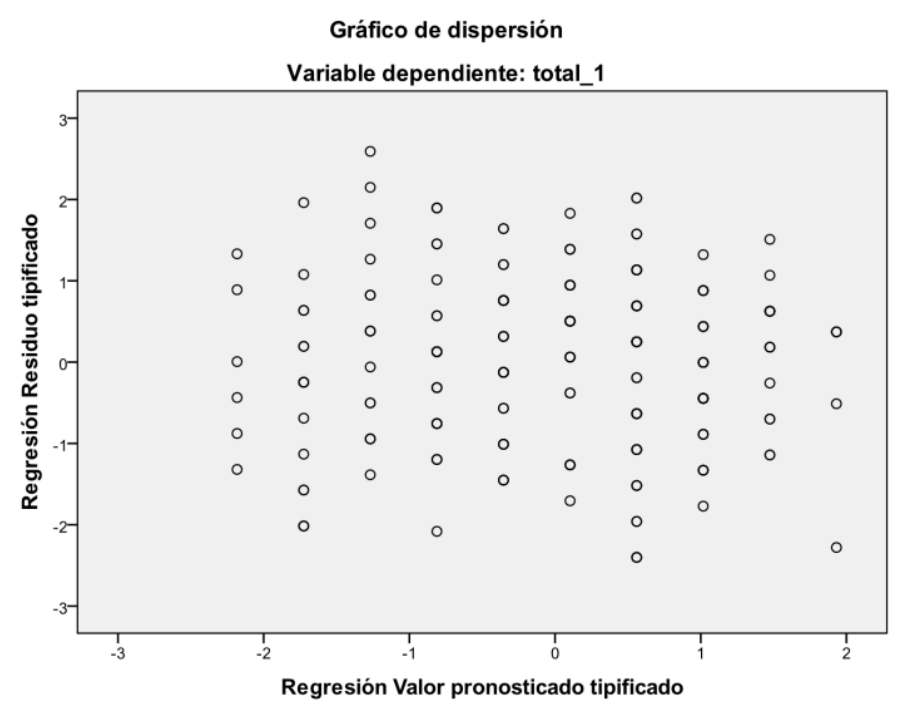

Gráfico 2. Análisis de residuos en la regresión lineal simple Fuente: Elaboración propia.

En la gráfica de dispersión (gráfico 3) observamos una relación muy elevada entre la puntuación total en PECO y en la versión abreviada, que cuantitativamente es de 0.928, tal y como aparece en el cuadro 5 .

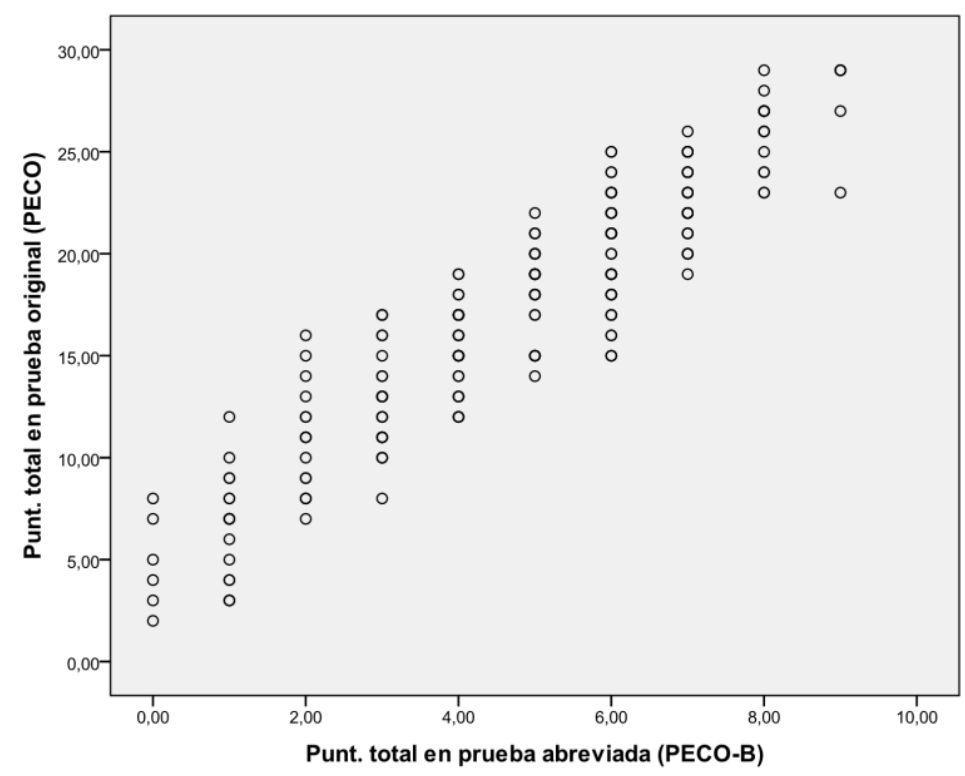

Gráfico 3. Relación entre PECO y versión abreviada Fuente: Elaboración propia.

Finalmente, por la sencillez del modelo predictivo y la utilidad para los usuarios, mostramos la tabla de equivalencias pronosticadas entre las PtA en la versión abreviada y en la prueba original PECO (cuadro 6). Además, teniendo en cuenta que cualquier modelo predictivo conlleva un margen de error, obtenemos un error máximo de estimación de 4,44 con un nivel de confianza del $95 \%$, que sumado y restado al valor 
pronosticado en la prueba original tendríamos el intervalo confidencial alrededor del cual se situaría la puntuación pronosticada.

Cuadro 6. Puntuaciones equivalentes pronosticadas en PECO a partir de la versión abreviada.

\begin{tabular}{ccc}
\hline $\begin{array}{c}\text { PUNT. TOTAL } \\
\text { ABREVIADA }\end{array}$ & $\begin{array}{c}\text { PUNT. TOTAL } \\
\text { PECO }\end{array}$ & $\begin{array}{c}\text { INTERPRETACIÓN } \\
\text { CUALITATIVA }\end{array}$ \\
\hline 9 & 28 & Nivel \\
8 & 26 & ALTO \\
7 & 23 & Nivel \\
\hline 6 & 20 & MEDIO \\
5 & 18 & Nivel \\
4 & 15 & BAJO \\
\hline 3 & 13 & \\
\hline & 10 & \\
\hline
\end{tabular}

Fuente: Elaboración propia.

De cara a la aplicación de esta versión abreviada, y para ser operativos, recomendamos aplicar esta versión teniendo en cuenta dos criterios. Por un lado, el nivel de dificultad de los ítems expresado por el índice de dificultad; y por otro, por las características de la tarea (identificación de fonemas-ítems 6, y 10-, adición de sílabas-ítems 13 y 14-, adición de fonemas -ítems 17 y 20-, omisión de sílabas -23 y 25-, omisión de fonemas - ítem 26-).

En el cuadro 6 se muestran los índices de dificultad de los nueve ítems que conforman la aplicación abreviada de PECO en el orden propuesto. La interpretación de la dificultad de los ítems se obtiene multiplicando por 100 cada uno de los ítems. Por ejemplo, el ítem "if6i" es resuelto correctamente por el $86 \%$ de los sujetos (muy fácil), mientras que el ítem "af20m" es resuelto correctamente por el $31 \%$ (difícil).

Cuadro 7. Análisis de dificultad de los ítems y orden de aplicación abreviada

\begin{tabular}{ccc}
\hline ORDEN DE APLICACIÓN & ÍTEM & ÍNDICE DE DIFICULTAD \\
\hline 1 & if6i & 0,86 \\
2 & if10m & 0,55 \\
3 & as $13 \mathrm{i}$ & 0,64 \\
4 & as $14 \mathrm{i}$ & 0,51 \\
5 & af $17 \mathrm{f}$ & 0,65 \\
6 & af20m & 0,31 \\
7 & os23i & 0,59 \\
8 & os25m & 0,21 \\
9 & of $26 \mathrm{i}$ & 0,44 \\
\hline
\end{tabular}

Fuente: Elaboración propia.

Para aplicar esta versión abreviada debe disponerse de la prueba original. Siguiendo el orden expuesto en el cuadro 7 , se dan al alumno básicamente las siguientes instrucciones:

- Ítem 6: De las siguientes palabras (se señalan los dibujos y se dicen las palabras) en qué palabra se oye el sonido /u/ (indio, saco, pie, uña, campana).

- Ítem 10: De las siguientes palabras (se señalan los dibujos y se dicen las palabras) en qué palabra se oye el sonido /r/ (avión, luna, vela, barco, zapato).

- Ítem 13: A la ficha blanca la llamaremos /mingo/ (la colocamos primero) mientras que la roja se llama /do/ (la colocamos delante), ¿qué palabra hemos formado?" 


\section{(domingo).}

- Ítem 14: A la ficha blanca la llamaremos /blado/ (la colocamos primero) mientras que la roja sigue llamándose /do/ (la colocamos delante), ¿qué palabra hemos formado? (doblado).

- Ítems 17: A esta ficha blanca la vamos a llamar / go/ (la colocamos en la mesa) y a esta ficha roja la seguimos llamando /1/, la colocamos detrás de la ficha blanca y decimos: "primero he puesto la ficha blanca que se llama /go/ y después la ficha roja que se llama $/ 1 /$, ¿qué palabra hemos formado? (gol)

- Ítem 20: Tenemos tres fichas. La ficha blanca se llamará /pe/ (la colocamos en la mesa). A la ficha amarilla la llamaremos /ado/ (la colocamos en la mesa ligeramente separado de la ficha blanca para dejar hueco a la ficha roja), y a esta ficha roja la llamando /1/, que colocamos en medio de las dos, ¿qué palabra hemos formado? (pelado).

- Ítem 23: Si a la palabra "saco” le quito el sonido /sa/, ¿cómo sonaría? (co).

- Ítem 25: Si a la palabra "gusano" le quito el sonido /sa/, (cómo sonaría) (gu...no).

- Ítem 26: Si a la palabra "foca” le quito el sonido /f/, ¿cómo sonaría? (oca).

Nos pareció interesante demostrar además, la validación de la versión abreviada y compararla con la validación en su versión completa. Para ello obtuvimos las siguientes evidencias tanto de validez concurrente como predictiva.

Para la validación concurrente se obtuvo la correlación de Pearson $\left(r_{\mathrm{xy}}\right)$ entre la PtA y los siguientes criterios externos en el mismo período temporal de la medición del conocimiento fonológico: 1) Valoración del maestro-a del "grado de capacidad del alumno para iniciar la lectoescritura” según una escala de 0 a 10;2) Lectura de palabras, y 3) Escritura de palabras.

Para obtener la validez predictiva se utilizaron dos criterios externos (comprensión lectora y escritura de palabras y pseudopalabras) que se correlacionaron con la PtA. Estos dos criterios fueron evaluados al año siguiente de haber aplicado la prueba; esto es, cuando los alumnos se encontraban en el tercer trimestre del primer curso de Educación Primaria. Para evaluar la comprensión lectora se utilizaron las pruebas de "estructuras sintácticas" y “comprensión de textos" de la batería PROLEC (Cuetos, Rodríguez y Ruano, 1996).

Por su parte, para evaluar la escritura de palabras y pseudopalabras se utilizaron las pruebas de "escritura de palabras", "escritura de pseudopalabras con sílabas complejas", "palabras de ortografía arbitraria" y "palabras con reglas ortográficas" de la Prueba de evaluación de la escritura en niños de Educación Primaria (Cuetos, Sánchez y Ramos, 1996). La puntuación obtenida en comprensión lectora fue la suma de la puntuación en estructuras sintácticas y comprensión de textos. En el caso de la escritura, se obtuvo una puntuación directa resultante de la suma de las cuatro pruebas de escritura.

En los cuedros 8 y 9 comprobamos que los resultados son bastantes aproximados entre la prueba original y la prueba abreviada. En todos los casos, los coeficientes de correlación son significativos, tanto en la validez concurrente como en la predictiva. 
Cuadro 8. Validez concurrente: correlación entre PECO y lectura, escritura y valoración del maestro

\begin{tabular}{lccc}
\hline & $\begin{array}{c}\text { LECTURA PALABRAS } \\
(\mathbf{N}=\mathbf{1 ~ 1 6})\end{array}$ & $\begin{array}{c}\text { ESCRITURA PALABRAS } \\
(\mathbf{N}=\mathbf{1 1 6})\end{array}$ & $\begin{array}{c}\text { VALORACIÓN } \\
\text { MAESTRO-A (N=240) }\end{array}$ \\
\hline PECO (abreviada) & $0,525 \mathrm{p}<0,001$ & $0,588 \mathrm{p}<0,001$ & $0,570 \mathrm{p}<0,001$ \\
PECO (completa) & $0,502 \mathrm{p}<0,001$ & $0,567 \mathrm{p}<0,001$ & $0,616 \mathrm{p}<0,001$ \\
\hline
\end{tabular}

Fuente: Elaboración propia.

Tabla 9. Validez predictiva: correlación entre PECO y comprensión lectora y escritura de palabras y pseudopalabras

\begin{tabular}{lcc}
\hline & $\begin{array}{c}\text { COMPRENSIÓN LECTORA } \\
\left(\mathbf{1}^{\circ} \text { PrIMARIA }\right)\end{array}$ & $\begin{array}{c}\text { ESCRITURA DE PALABRAS Y } \\
\text { PSEUDOPALABRAS } \\
\left(\mathbf{1}^{\circ} \text { PRIMARIA }\right)\end{array}$ \\
\hline PECO (abreviada) & $0,374 \mathrm{p}<0,001$ & $0,258 \mathrm{p}<0,001$ \\
PECO (completa) & $0,395 \mathrm{p}<0,001$ & $0,304 \mathrm{p}<0,001$ \\
\hline
\end{tabular}

Fuente: Elaboración propia.

En síntesis, a partir de los resultados obtenidos obtuvimos una prueba abreviada y válida del conocimiento fonológico a partir de la prueba original PECO que nos permitirá disponer de un instrumento de evaluación y de investigación de un aspecto tan importante como es el conocimiento fonológico en la adquisición del principio alfabético y en los momentos iniciales del aprendizaje lectoescritor.

\section{Discusión y conclusiones}

Este trabajo aporta una versión abreviada de PECO que constituye una alternativa para la detección de las dificultades en conocimiento fonológico, si tenemos en cuenta que podríamos reducir el tiempo de aplicación a una tercera parte de la aplicación completa. Es decir, la aplicación abreviada se realizaría en 10-12 minutos.

El conjunto de ítems obtenidos en el modelo de regresión constituye una óptima representación de lo que denominamos conocimiento fonológico, puesto que se obtiene una versión no homogénea de ítems (tal y como es considerado teóricamente el constructo "conocimiento fonológico") y resulta de enorme interés en la evaluación de aquellas variables explicativas de las dificultades de lectura y escritura relacionadas con los procesos fonológicos.

Estamos seguros que, tanto los profesionales con responsabilidad en detectar lo antes posibles las dificultades de lectura y escritura (orientadores, logopedas, psicólogos, pedagogos, etc.) como los investigadores, dispondrán en la versión abreviada de PECO de un adecuado instrumento para evaluar el conocimiento fonológico.

Por otro lado, dada la sencillez de aplicación y la importancia que posee el conocimiento fonológico en los momentos iniciales de la lectura y la escritura, la aplicación abreviada de PECO podría formar parte del conjunto de pruebas que se aplican al finalizar la educación infantil con la finalidad de predecir dificultades lectoescritoras, especialmente de tipo disléxico, y promover las medidas preventivas de refuerzo y apoyo más adecuadas. 


\section{Referencias}

Bravo, L., Villalón, M. y Orellana, E. (2011). La conciencia fonológica y la lectura inicial en niños que ingresan a primer año básico. Psykhe, $11(1)$.

Byrne, B. y Fielding-Barnsley, R. (1989). Phonemic awareness and letter knowledge in the child's acquisition of the alphabetic principle. Journal of Educational Psychology, 81(3), 313-321. https://doi.org/10.1037/0022-0663.81.3.313

Carrillo, M. y Sánchez, J. (1991). Segmentación fonológico-silábica y adquisición de la lectura: un estudio empírico. Comunicación, Lenguaje y Educación, 9, 109-116.

Carrillo, M. y Sánchez, J. (1996). Desarrollo metafonológico y adquisición de la lectura: un programa de entrenamiento. Madrid: MEC-CIDE.

Cuetos, F., Sánchez, C. y Ramos, J. L. (1996). Evaluación de los procesos de escritura en niños de educación primaria. Bordón, 48(4), 445-465.

Cuetos, F., Rodríguez, B. y Ruano, E. (1996). Evaluación de los Procesos Lectores (PROLEC). Madrid: TEA ediciones.

Cutting, L. E. y Denckla, M. B. (2001). The relationship of rapid serial naming and Word reading in normally developing readers: An exploratory model. Reading and Writing, 14(7-8), 673705.

De Jong, P. F. y Olson, R. K. (2004). Early predictors of letter knowledge. Journal of Experimental Child Psychology, 88(3), 254-273. https://doi.org/10.1016/j.jecp.2004.03.007

De Jong, P. F. y Van der Leij, A. (1999). Specific contributions of phonological abilities to early reading acquisition: Results from a Dutch latent variable longitudinal study. Journal of Educational Psychology, 91(3), 450-476. https://doi.org/10.1037/0022-0663.91.3.450

Defior, S. y Serrano, F. (2011). Procesos fonológicos explícitos e implícitos, lectura y dislexia. Revista Neuropsicología, Neuropsiquiatría y Neurociencias, 1, 79-94.

Delgado, M. y Sancho, M. S. (2014). Prerrequisitos para el proceso de aprendizaje de la lectura y la escritura: conciencia fonológica y destrezas orales de la lengua. Lengua y Habla, 18, 7292.

Domínguez, A. B. y Clemente, M. (1993). ¿Cómo desarrollar secuencialmente el conocimiento fonológico? Comunicación, Lenguaje y Educación, 19-20, 171-181.

Domínguez, A. B. (1992). La enseñanza de las habilidades de análisis fonológico en el aprendizaje de la lectura y de la escritura. Programas para la Educación Infantil. Tesis doctoral. Universidad de Salamanca.

Domínguez, A. B. (1996). Evaluación de los efectos a largo plazo de la enseñanza de habilidades de análisis fonológico en el aprendizaje de la lectura y la escritura. Infancia y Aprendizaje, 76, 83-96.

Feld, V. (2014). Las habilidades fonológicas, su organización neurofisiológica y su aplicación en la educación. Pensamiento Psicológico, 12(1), 71-82.

Gómez, F. R., González, A., Zarabozo, D. y Amano, M. (2010). La velocidad de denominación de letras. Revista Mexicana de Investigación Educativa, 15(46), 823-848.

González, R. M., Cuetos, F., Vilar, J. y Uceira, E. (2015). Efectos de la intervención en conciencia fonológica y velocidad de denominación sobre el aprendizaje de la escritura. Aula abierta, 43, $1-8$. 
Guzmán, R., Jiménez, J., Ortiz, M., Hernández-Valle, I., Estévez, A., Rodrigo, M., García, E., Díaz, A. y Hernández, S. (2004). Evaluación de la velocidad de nombrar en las dificultades de aprendizaje de la lectura. Psicothema, 16, 442-447.

Jiménez, J. y Ortiz, M. R. (1995). Conciencia fonológica y aprendizaje de la lectura: teoría, evaluación e intervención. Madrid: Síntesis.

Kirby, J. R., Parrila, R. K. y Pfeiffer, S. L. (2003). Naming Speed and Phonological Awareness as Predictors of Reading Development. Journal of Educational Psychology, 95(3), 453-464.

Leal, F. y Suro, J. (2012). Las tareas de conciencia fonológica en preescolar: una revisión de las pruebas empleadas en población hispanohablante. Revista Mexicana de Investigación Educativa, 17(54), 729-757.

López-Escribano, C. (2007). Contribuciones de la neurociencia al diagnóstico y tratamiento educativo de la dislexia del desarrollo. Revista de Neurología, 44(3), 173-180.

Manis, F. R., Seidenberg, M. S. y Doi, L. M. (1999). See Dick RAN: Rapid Naming and the Longitudinal Prediction of Reading Subskills in First and Second Graders. Scientific Studies of Reading, 3(2), 129-157. https://doi.org/10.1207/s1532799xssro302_3

Muñoz, C. (2011). Aprendizaje de la lectura y conciencia fonológica: Un enfoque psicolingüístico del proceso de alfabetización inicial. Psykhe, $11(1)$.

Ortiz, M. R. (1994). Conciencia fonológica y conciencia del lenguaje escrito en el aprendizaje de la lectura. Tesis doctoral. Universidad de La Laguna.

Powell, D., Stainthorp, R., Stuart, M., Garwood, H. y Quinlan, P. (2007). An experimental comparison between rival theories of rapid automatized naming performance and its relationship to reading. Journal of Experimental Child Psychology, 98(1), 46-68. https://doi.org/10.1016/j.jecp.2007.04.003

Ramos, J. L. y Cuadrado, I. (2004). Influence of spoken language on initial acquisition of reading/writing: critical analysis of verbal deficit theory. Reading Psychology, 25(3), 149165 .

Ramos, J. L. y Cuadrado, I. (2006a). Prueba para la Evaluación del Conocimiento Fonológico - PECO. Madrid: EOS.

Ramos, J. L. y Cuadrado, I. (2006b). Cuaderno para el entrenamiento y refuerzo en conocimiento fonológico. Madrid: EOS.

Ramos, J. L. (2000). Entrenamiento en tareas de conocimiento fonológico y su influencia en la adquisición inicial de la lectura y la escritura. Ciencia Psicológica, 7, 64-82.

Ramos, J. L. (2004). Conocimiento fonológico y desarrollo lectoescritor en educación infantil. Educación XXI, 7, 169-183.

Sánchez, E., Rueda, M. I. y Orrantía, J. (1989). Estrategias de intervención para la reeducación de niños con dificultades en al aprendizaje de la lectura y de la escritura. Infancia y Aprendizaje, 3-4, 101-111.

Sebastián, M. E. y Maldonado, A. (1987). La segmentación de palabras: un prerrequisito del aprendizaje de la lectura. Boletín I.C.E. Madrid: Universidad Autónoma de Madrid.

Suárez, P., García, M. y Cuetos, F. (2013). Variables predictoras de la lectura y la escritura en castellano. Infancia y Aprendizaje, 36, 77-89. 
Treiman, R. (1991). The role of intrasyllabic units in learning to read. En L. Rieben y C.A. Perfetti (Eds.), Learning to read: basic research and its implications (pp. 149-160). Hillsdale: Lawrence Erlbaum Associates.

Trias, D., Cuadro, A. y Costa, D. (2009). Desarrollo de la conciencia fonémica: evaluación de un programa de intervención. Ciencias Psicológicas, $\operatorname{III}(2), 177-184$.

Vellutino, F. R., Fletcher, J. M., Snowling, M. J. y Scanlon, D. M. (2004). Specific Reading disability (dyslexia): what have we learned in the past four decades? Journal of Child Psychology and Psychiatry, 45(1), 2-40. https://doi.org/10.1046/j.0021-9630.2003.00305.x

Wagner, R. K., Torgesen, J. K. y Rashotte, C. A. (1994). Development of reading-related phonological processing abilities: New evidence of bidirectional causality from a latent variable longitudinal study. Developmental Psychology, 30(1), 73-87.

Yopp, H. (1988). The validity and reliability of phonemic awareness test. Reading Research Quarterly, 23, 159-177.

\section{Breve CV de los autores}

\section{José Luís Ramos Sánchez}

Licenciado en Ciencias de la Educación por la UNED y doctor en Pedagogía por la Universidad de Extremadura. Máster en Métodos Actuales de Estadística Aplicada. Profesor Asociado en el área de Métodos de Investigación y Diagnóstico en Educación de la Universidad de Extremadura. Acreditado Profesor Titular de Universidad por la ANECA desde 2009. Desde 1988 es orientador educativo y ha sido Jefe del Servicio de Programas Educativos y Atención a la Diversidad de la Consejería de Educación (Junta de Extremadura). Pertenece al Grupo de Investigación e Innovación Educativa de Extremadura (GRIE) de la UEX. Director de tesis doctorales y trabajos de investigación. Ha participado en proyectos de investigación nacionales y europeos. Autor y coautor de más de cincuenta artículos en revistas científicas nacionales e internacionales y capítulos de libros y libros. Entre sus publicaciones destacan las relacionadas con los métodos de investigación y el análisis de datos en la investigación educativa y social, la evaluación del desarrollo del lenguaje, la conciencia fonológica, la intervención psicopedagógica, evaluación e intervención ante las dificultades de aprendizaje, y en general sobre los procesos de lectura y escritura. ORCID ID: 0000-0002-6636-9383. Email:jlramos@unex.es

\section{Ana Isabel González Contreras}

Licenciada en Psicología por la Universidad de Salamanca y doctora en Psicología por la Universidad de Extremadura. Ttulada en psicología clínica por el MEC. Diplomada en profesorado de EGB. Máster Universitario de Investigación en Ciencias Sociales y Jurídicas y Máster en Pedagogía Terapéutica. Profesora asociada del departamento de Psicología y Antropología del área de Psicología Social de la Universidad de Extremadura. Sus líneas de investigación están relacionadas con la convivencia escolar, las dificultades de aprendizaje y el desarrollo socio-familiar. Ha presentado comunicaciones en congresos nacionales e internacionales. En el ámbito privado, ejerce como psicóloga clínica y Revista Iberoamericana de Evaluación Educativa 15 educativa. Asimismo, forma parte del turno de 
peritaciones judiciales del Colegio Oficial de Psicólogos de Extremadura y de profesionales de la Psicología para la emisión de informes periciales en el ámbito de actuación de tribunales y juzgados en el territorio español. ORCID ID: 0000-0001-87146325. Email: anaisabelgc@unex.es 